\title{
Comparing the Effectiveness of Behavioral Activation and Acceptance and Commitment Therapy in Cognitive-Attentional Syndrome in Patients with Depression
}

\author{
Sahar Amiri ${ }^{1}$, Parvin Ehteshamzadeh (10 ${ }^{1,}{ }^{*}$, Fariba Hafezi ${ }^{1}$ and Mohammad Reza Borna ${ }^{1}$ \\ ${ }^{1}$ Department of Psychology, Ahvaz Branch, Islamic Azad University, Ahvaz, Iran \\ "Corresponding author: Department of Psychology, Ahvaz Branch, Islamic Azad University, Ahvaz, Iran. Email: ehteshamzadehp@gmail.com
}

Received 2020 September 19; Revised 2021 February 06; Accepted 2021 February 06.

\begin{abstract}
Background: Cognitive-attentional syndrome(CAS) is one of the primary constructs of depression, the treatment of which is highly challenging because of its high prevalence and the emergence of symptoms such as feelings of sadness, emptiness, and hopelessness.

Objectives: The present study aimed to investigate the effectiveness of behavioral activation (BA) and acceptance and commitment therapy (ACT) in CAS in patients with depression.

Methods: This quasi-experimental research utilized a pretest, posttest, and two-month follow-up design with a control group. The study population comprised 260 patients with a diagnosis of depression who referred to the psychological centers of Ahvaz in 2019. The sample consisted of 45 patients with depression selected by convenience sampling. We randomly divided the participants into two experimental groups (BA and ACT) and a control group ( $n=15$ per group). The research instrument included the cognitiveattentional syndrome (CAS-1) questionnaire. Data were analyzed using SPSS version 24.0 with descriptive and inferential statistics, such as mean, standard deviation, and multivariate analysis of covariance (MANCOVA).

Results: In terms of CAS and its components, there was a significant difference between the experimental and control groups in the posttest and follow-up phases $(\mathrm{P}=0.0001)$. Besides, ACT and BA significantly reduced CAS and its components in the posttest phase $(\mathrm{P}=0.0001)$. However, the effectiveness of $\mathrm{BA}$ in decreasing CAS and its components remained during the follow-up phase. Furthermore, the results showed that ACT was more effective in reducing attention to threat and metacognitive beliefs during the posttest phase.
\end{abstract}

Conclusions: According to research findings, both BA and ACT are efficient therapies in reducing CAS in patients with depression. Thus, both approaches can be used to strengthen treatment interventions to reduce CAS in patients with depression.

Keywords: Behavior, Acceptance and Commitment Therapy, Cognitive, Depression

\section{Background}

Depression is a mental disorder with symptoms such as sadness, emptiness, hopelessness, and decreased interest or enjoyment in most daily activities. The disorder is associated with physical symptoms such as headache, fatigue, anorexia, insomnia, constipation, hypotension, etc (1). On the other hand, patients suffering from depression have frequently self-reported negative feelings (2) and lack of control over thoughts and behaviors (3). The last symptom is associated with the recurrent activation of the cognitive-attentional syndrome (CAS) (2), which is one of the primary constructs in the metacognitive theory of emotional disorders. The syndrome encompasses a set of psychological processes such as recurrent negative thoughts (concern and rumination), threat-focused attention, and cognitive-behavioral strategies (4).

According to many studies, CAS is a significant factor in developing mood disorders, including depressive disorder, anxiety disorder, post-traumatic stress disorder, and obsessive-compulsive disorder $(4,5)$. Cognitiveattentional syndrome makes individuals suffer from distorted and biased thoughts, which are out of control. This would consequently make their emotional problems permanent and intensified (6). In other words, this syndrome leads to the adoption of self-regulatory strategies or maladaptive coping behaviors, which are considered useful by the same individual; however, they have opposite effects and make emotional problems last longer (6). Cognitive-attentional syndrome is rooted in the metacog- 
nitive approach to psychological disorders (7), the removal of which is essential by assisting the patients to detect new approaches to control attention, link to negative thoughts and beliefs, and change metacognitive beliefs creating maladaptive thinking patterns (5). In this regard, behavioral activation and acceptance and commitment therapy are the two main treatments derived from clinical behavior analysis and metacognitive approaches to depression (8).

Behavioral activation (BA) is a type of nonpharmacological intervention based on Skinner's psychological model of behavior change, known as functional behavioral assessment (9). Behavioral activation is underpinned by an assumption indicating that the lack of adequate environmental support or excessive environmental punishment arouse depression. The intervention aims to promote environmental reinforcement and decrease environmental punishment (10). Jafari et al. (11) showed that BA is effective in improving depressive symptoms and quality of life by helping the individual to distance himself from the psychological processes of accreditation to painful emotions. Timurpour et al. (12) showed that behavioral activation therapy is significantly effective in increasing cognitive flexibility and emotional resilience in patients with depressive disorder. Acceptance and commitment therapy (ACT) is also one of the third-wave psychological therapies considered not only in the field of positive psychology but also in the treatment of psychological damages and disorders such as depression and anxiety. It is based on mindfulness (13), i.e., the conscious involvement of awareness along with openness, interest, and acceptance in one's here-and-now experiences(14). In this regard, when individuals view their personal experiences (i.e., thoughts and feelings) with openness and acceptance, even the most painful of which seems to be less threatening and more tolerable (15).

Previous studies have revealed the effectiveness of BA in decreasing depression. For example, Kellett et al. (16) reported that behavioral activation can be an effective depression treatment option that shows some clinical promise. Szuhany et al. (17) reported that BA may be a useful strategy for improving depression and increasing exercise. Babakhanzadeh et al. (18) showed that cognitive therapy, with emphasis on executive functions, reduced cognitive-attention syndrome, and improved the sense of coherence among students. Melli et al. (19) reported that metacognition may have an important role in health anxiety in clinical samples. The effectiveness of ACT in decreasing depressive symptoms was also confirmed $(20,21)$. A-Tjak et al. (20) showed that ACT, as well as reductions in avoidant and anxious attachment, predicts symptom reduction after psychological treatment for depression. Kazemeyni et al. (21) showed that ACT significantly reduced the rate of depression and increased psychological flexibility in women with depression.

However, it is not known which intervention can have the highest positive effects on CAS in individuals suffering from depression. Given that depression is one of the most common psychological problems that is associated with huge costs at the individual and social level, it is necessary to take effective interventions and basic steps to prevent and treat psychological problems. Identifying the best and most cost-effective treatment is also essential to reduce disease rates and treatment.

\section{Objectives}

The present study aimed to compare the effectiveness of behavioral activation and acceptance and commitment therapy on the cognitive-attentional syndrome in patients with depression in Ahvaz city.

\section{Methods}

This quasi-experimental research utilized a pretest, posttest, and follow-up design with a control group. The study population comprised 260 patients with a diagnosis of depression who referred to the psychological centers of Ahvaz in 2019.

Forty-five patients with depression were selected as the sample of the study using convenience sampling. Fifteen participants were included in each group using $G^{*}$ power statistical software with an effect size of 1.8 , a test power of 0.95 , and $\alpha=0.05$. Randomization was carried out by the researcher, and participants were allocated by the cointhrowing method. Randomization was undertaken after consent to participate and completion of all the baseline measures and eligibility interviews. Participants were randomly divided into two experimental groups (BA and ACT) and a control group ( $\mathrm{n}=15$ per group).

The inclusion criteria were a diagnosis of moderate depression, a score of 20 to 28 on the Beck depression inventory (BDI), voluntary participation in non-drug treatment, and no use of medications or any type of treatment intervention during the study. The exclusion criteria were absence from more than one treatment session and reluctance to continue the treatment process. After sampling, the intervention programs were performed on the experimental groups. The control group did not receive any treatment. The intervention programs in the experimental groups were conducted in parallel in the evenings by the first author in the Peyvand Psychology and Counseling Center in Ahvaz. 
After the intervention sessions, a posttest was performed in the experimental and control groups. A follow-up was further conducted in both groups after two months. At the end of the study, to observe ethical considerations, the control group received a course of BA and ACT. For ethical considerations, the researchers received written consent from the participants for participation in the research. The study was approved by the Ethics Committee of Islamic Azad University, Ahvaz Branch (code: IR.IAU.AHVAZ.REC.1399.009).

\subsection{Research Instruments}

Cognitive-attentional syndrome (CAS-1) questionnaire: This 16-item questionnaire developed by Wells (5) encompasses three components: concern and threat (items 1 and 2), avoidant coping (items 3-8), and metacognitive beliefs (items 9-16). The first eight items are scored based on a nine-point Likert scale ranging from 0 to 8 , and the second eight items assess individuals' beliefs in each of the metacognitive beliefs about CAS on a 0 - 100 scoring scale. The total CAS score is obtained from all the 16 items and ranges from 0 to 858, with a higher score indicating more CAS. Salmani and Hasani (22) reported a Cronbach's alpha coefficient of 0.85 for the whole questionnaire. In the present study, Cronbach's alpha coefficient was 0.78 for the questionnaire.

Beck depression inventory: Beck's depression inventory was developed by Beck and contains 25 items. Each item has four options scored based on a scale ranging from 0 to 3 , indicating different degrees of depression from mild to severe. The minimum and maximum scores are zero and 75 , respectively. In this regard, scores 0 - 13, 14 - 19, 20 - 28, and 29 - 75 represent minimal, mild, moderate, and severe depression, respectively. In the present study, Cronbach's alpha coefficient was 0.84 for the questionnaire.

\subsection{Intervention Program}

The first intervention program consisted of eight 90min sessions of BA therapy (23). The second intervention program consisted of eight 60-min sessions of ACT (24). Table 1 presents a summary of the sessions.

\subsection{Statistical Analyses}

Data were analyzed by descriptive and inferential statistics, such as mean, standard deviation, and multivariate analysis of covariance (MANCOVA). The Bonferroni post hoc test was utilized to investigate the difference in the means between the pretest, posttest, and paired follow-up using SPSS version 24.0.

\section{Results}

According to the descriptive statistics, the participants in the experimental and control groups were in the age range of 30 - 60 years. The demographic variables of the participants are shown in Table 2.

Table 3 presents the mean and standard deviation (SD) of the pretest, posttest, and follow-up scores of concerns and threat, avoidant coping, metacognitive beliefs, and cognitive-attentional syndrome, for the experimental and control groups.

To test the normality of the collected data concerning the significance of the Z-value, the Kolmogorov-Smirnov test revealed that the research variables had a normal distribution. To test the homogeneity of variances (for the same variances of the experimental and control groups), Levene's test was used. Due to the homogeneity of the variances, the analysis of covariance was used to analyze the data. As shown in Tables 4 and 5, while controlling the pretest effect, there was a significant difference between at least one of the BA and ACT experimental groups and the control group in the posttest and follow-up phases (P $<0.05)$.

Table 6 also shows significant differences in the posttest phase between the experimental groups and the control group in terms of all the CAS subscales ( $P$ $<0.05)$. In this regard, ACT was more effective than BA on the overall reduction of CAS and the components of concern and threat and metacognitive beliefs $(\mathrm{P}<0.05)$. Although no significant difference existed between the effects of the two interventions on the avoidant coping strategies $(\mathrm{P}>0.05)$, only during the two-month follow-up phase, the mean differences between the BA group and the control group were significant in terms of the CAS scale and its three components $(\mathrm{P}<0.05)$. In other words, in the follow-up phase, the effectiveness of BA treatment in reducing CAS was still observed; however, the effectiveness of ACT treatment lasted for none of the CAS components over time.

\section{Discussion}

According to the findings, both BA and ACT decreased the three main CAS components immediately following the intervention program. Although there was significantly higher effectiveness for the ACT technique than for BA, the effectiveness of the BA technique was still observed over time, as it reduced all cognitive symptoms even after a two-month interval. This finding is consistent with the research results of Babakhanzadeh et al. (18), Melli et al. (19), and A-Tjak et al. (20). 


\begin{tabular}{|c|c|c|}
\hline Sessions & Content of BA Therapy Sessions & Content of ACT Sessions \\
\hline First & $\begin{array}{l}\text { Communicating and introducing members, stating objectives, } \\
\text { introducing the course, and behavioral contracts }\end{array}$ & $\begin{array}{l}\text { Introducing members, mentioning group regulations and objectives, and } \\
\text { introducing the course }\end{array}$ \\
\hline Second & $\begin{array}{l}\text { Focusing behavioral activation on the interaction between individuals and } \\
\text { the environment/Detecting and changing overt behaviors }\end{array}$ & $\begin{array}{l}\text { Introducing some concepts of ACT, including the experience of avoidance, } \\
\text { integration, and mental acceptance }\end{array}$ \\
\hline Third & $\begin{array}{l}\text { Providing psychological programs for group healing processes with a } \\
\text { focus on four subjects: justification of the general context of the disease, } \\
\text { selection of appropriate treatment methods, individual's status, and } \\
\text { motivational issues }\end{array}$ & $\begin{array}{l}\text { Implementing ACT techniques such as cognitive isolation, psychological } \\
\text { awareness, and self-visualization }\end{array}$ \\
\hline Fourth & $\begin{array}{l}\text { Focusing on different dimensions of stress and anxiety, using positive } \\
\text { verbal reinforcement by hope therapy (i.e., the use of positive verbal } \\
\text { reinforcement by expressing positive sentences for each other and hoping } \\
\text { for small improvements in therapies) }\end{array}$ & $\begin{array}{l}\text { Teaching treatment techniques, emotional awareness, and wise awareness } \\
\text { (self-victim metaphor) }\end{array}$ \\
\hline Fifth & $\begin{array}{l}\text { Focusing on different dimensions of depression and psychological and } \\
\text { mood changes by using allegory }\end{array}$ & $\begin{array}{l}\text { Teaching self-treatment techniques as a background and practice. } \\
\text { Mindfulness techniques and distress tolerance training }\end{array}$ \\
\hline Sixth & $\begin{array}{l}\text { Focusing on behavioral modes (requirements for relief from anxiety and } \\
\text { depression) and cognitive judgments and their control (i.e., an ability to } \\
\text { solve problems and relieve stress and the use of emotion-based coping) }\end{array}$ & $\begin{array}{l}\text { Teaching treatment techniques of personal values and clarifying values, } \\
\text { teaching emotion regulation/(bad cup metaphor) }\end{array}$ \\
\hline Seventh & $\begin{array}{l}\text { Educating individuals about the psychological and social components of } \\
\text { the existing situation, teaching coping skills and behavioral reconstruction }\end{array}$ & $\begin{array}{l}\text { Teaching treatment techniques of personal values and committed action } \\
\text { and promoting interpersonal efficiency (chess scene metaphor) }\end{array}$ \\
\hline Eighth & $\begin{array}{l}\text { Providing a summary of treatment, follow-up solutions, and feedback by } \\
\text { the group members }\end{array}$ & $\begin{array}{l}\text { Reviewing and practicing the trained treatment techniques with an } \\
\text { emphasis on regulating emotions and a sense of meaningfulness in real } \\
\text { world }\end{array}$ \\
\hline
\end{tabular}

Abbreviations: BA, behavioral activation; ACT, acceptance and commitment therapy

\begin{tabular}{|c|c|c|c|c|}
\hline Groups & BA & ACT & Control & Total \\
\hline \multicolumn{5}{|l|}{$\operatorname{Age}(y)$} \\
\hline $30-40$ & $7(23.33)$ & $9(30.00)$ & $7(23.33)$ & $23(51.11)$ \\
\hline $40-50$ & $6(20.00)$ & $4(13.33)$ & $5(16.67)$ & $15(33.33)$ \\
\hline $50-60$ & $2(6.67)$ & $2(6.67)$ & $3(10.00)$ & $7(15.56)$ \\
\hline \multicolumn{5}{|l|}{ Gender } \\
\hline Male & $11(36.67)$ & $11(36.67)$ & $12(40.00)$ & $34(75.56)$ \\
\hline Female & $4(13.33)$ & $4(13.33)$ & $3(10.00)$ & $11(24.44)$ \\
\hline \multicolumn{5}{|l|}{ Marital status } \\
\hline Married & $10(33.33$ & $8(26.67)$ & $9(30.00)$ & $27(60.00)$ \\
\hline Single & $5(16.67)$ & $7(23.33)$ & $6(20.00)$ & $18(40.00)$ \\
\hline
\end{tabular}

Abbreviations: BA, behavioral activation; ACT, acceptance and commitment therapy.

${ }^{\mathrm{a}}$ Values are expressed as No. (\%).

Behavioral activation teaches patients to become aware of their anxiety and threatening senses and reach them by evaluating behaviors and moods, selecting alternative responses, undertaking alternative responses, integrating the alternatives, and observing and assessing their consequences (23). It also makes them achieve alternative responses instead of temporary avoidance, such as depression, isolation, and so on, by solving problems and adopting positive emotion-oriented coping strategies, with whom they control their CAS. On the other hand, ACT also teaches skills such as living in the present, considering and focusing on recent experiences, strengthening emotional awareness, accepting negative emotions and thoughts without prejudice and judgment (15), reducing the frequency of negative emotions and thoughts such as rumination and concerns, and raising hypervigilance; thereby, this technique disrupts the CAS cycle.

Needless to note that the cycle of CAS-induced dysfunctional and temporary coping is exacerbated by the long-term reflection of inappropriate metacognitive be- 


\begin{tabular}{|c|c|c|c|}
\hline Dependent Variable & BA & ACT & Control \\
\hline \multicolumn{4}{|l|}{ Concerns and threat } \\
\hline Pre-test & $12.86 \pm 1.64$ & $12.20 \pm 1.26$ & $12.93 \pm 1.22$ \\
\hline Post-test & $8.40 \pm 1.54$ & $6.20 \pm 1.08$ & $12.73 \pm 1.03$ \\
\hline Follow-up & $8.53 \pm 1.53$ & $12.20 \pm 1.72$ & $12.86 \pm 1.48$ \\
\hline \multicolumn{4}{|l|}{ Avoidant coping } \\
\hline Pre-test & $37.86 \pm 1.38$ & $37.80 \pm 1.35$ & $38.13 \pm 1.53$ \\
\hline Post-test & $21.46 \pm 1.59$ & $22.80 \pm 1.82$ & $37.53 \pm 2.55$ \\
\hline Follow-up & $21.60 \pm 1.63$ & $37.53 \pm 2.13$ & $37.86 \pm 2.55$ \\
\hline \multicolumn{4}{|l|}{ Metacognitive beliefs } \\
\hline Pre-test & $568.53 \pm 25.13$ & $574.00 \pm 13.75$ & $567.86 \pm 15.08$ \\
\hline Post-test & $317.26 \pm 19.16$ & $221.26 \pm 17.81$ & $562.46 \pm 19.80$ \\
\hline Follow-up & $317.53 \pm 9.76$ & $572.86 \pm 14.22$ & $568.53 \pm 25.13$ \\
\hline \multicolumn{4}{|c|}{ Cognitive-attentional syndrome } \\
\hline Pre-test & $619.26 \pm 27.41$ & $624.00 \pm 13.79$ & $618.93 \pm 17.15$ \\
\hline Post-test & $347.13 \pm 9.06$ & $250.26 \pm 18.02$ & $612.73 \pm 19.46$ \\
\hline Follow-up & $347.66 \pm 9.68$ & $622.60 \pm 14.29$ & $619.26 \pm 27.41$ \\
\hline
\end{tabular}

Abbreviations: BA, behavioral activation; ACT, acceptance and commitment therapy; M, mean; SD, standard deviation.

${ }^{a}$ Values are expressed as mean $\pm(\mathrm{SD})$.

\begin{tabular}{|c|c|c|c|c|c|c|}
\hline Variable & SS & df & MS & $\mathbf{F}$ & $\mathbf{P}$ & $\eta \mathbf{p 2}$ \\
\hline \multicolumn{7}{|c|}{ Concerns and threat } \\
\hline Pre-test & 1.47 & 1 & 1.47 & 0.95 & 0.335 & 0.02 \\
\hline Group & 310.81 & 2 & 155.40 & 100.40 & 0.000 & 0.83 \\
\hline \multicolumn{7}{|c|}{ Avoidant coping } \\
\hline Pre-test & 11.82 & 1 & 11.82 & 2.99 & 0.091 & 0.06 \\
\hline Group & 2356.22 & 2 & 1178.11 & 298.09 & 0.0001 & 0.093 \\
\hline Error & 162.03 & 41 & 3.492 & & & \\
\hline \multicolumn{7}{|c|}{ Metacognitive beliefs } \\
\hline Pre-test & 11.05 .95 & 1 & 1105.95 & 4.53 & 0.03 & 0.10 \\
\hline Group & 923397.35 & 2 & 461698.67 & 1892.27 & 0.0001 & 0.98 \\
\hline Error & 10003.64 & 41 & 243.99 & & & \\
\hline \multicolumn{7}{|c|}{ Cognitive-attentional syndrome } \\
\hline Pre-test & 1294.94 & 1 & 1294.94 & 5.47 & 0.024 & 0.11 \\
\hline Group & 1054897.42 & 2 & 527448.71 & 2228.35 & 0.0001 & 0.99 \\
\hline Error & 9704.66 & 41 & 236.69 & & & \\
\hline
\end{tabular}

Abbreviations: SS, sum of squares; df, degrees of freedom; MS, mean squares; $\eta$ p2, partial eta-squared. 


\begin{tabular}{|c|c|c|c|c|c|c|}
\hline Variable & SS & df & MS & $\mathbf{F}$ & $\mathbf{P}$ & $\eta \mathbf{p} 2$ \\
\hline \multicolumn{7}{|c|}{ Concerns and threat } \\
\hline Pre-test & 25.35 & 1 & 25.35 & 15.62 & 0.0001 & 0.27 \\
\hline Group & 172.76 & 2 & 86.37 & 53.24 & 0.0001 & 0.72 \\
\hline Error & 66.51 & 41 & 1.62 & & & \\
\hline \multicolumn{7}{|c|}{ Avoidant coping } \\
\hline Pre-test & 108.15 & 1 & 108.15 & 52.22 & 0.0001 & 0.56 \\
\hline Group & 2569.50 & 2 & 1284.75 & 620.34 & 0.0001 & 0.96 \\
\hline Error & 84.91 & 41 & 2.07 & & & \\
\hline \multicolumn{7}{|c|}{ Metacognitive beliefs } \\
\hline Pre-test & 1989.43 & 1 & 1989.43 & 7.29 & 0.01 & 0.15 \\
\hline Group & 633982.89 & 2 & 316991.44 & 1178.60 & 0.0001 & 0.98 \\
\hline Error & 11023.76 & 41 & 268.87 & & & \\
\hline \multicolumn{7}{|c|}{ Cognitive-attentional syndrome } \\
\hline Pre-test & 2872.16 & 1 & 2872.16 & 9.95 & 0.003 & 0.19 \\
\hline Group & 739789.66 & 2 & 369894.83 & 1282.21 & 0.0001 & 0.98 \\
\hline Error & 11827.70 & 41 & 288.48 & & & \\
\hline
\end{tabular}

Abbreviations: SS, sum of squares; df, degrees of freedom; MS, mean squares; $\eta \mathrm{p} 2$, partial eta-squared.

\begin{tabular}{|c|c|c|c|c|}
\hline \multirow{2}{*}{ Variable } & \multicolumn{2}{|c|}{ Posttest } & \multicolumn{2}{|c|}{ Follow-Up } \\
\hline & $\begin{array}{c}\text { Mean Difference (95\% } \\
\text { CI) }\end{array}$ & $\mathbf{P}$ & $\begin{array}{l}\text { Mean Difference (95\% } \\
\text { CI) }\end{array}$ & $\mathbf{P}$ \\
\hline \multicolumn{5}{|l|}{ Concerns and threat } \\
\hline Ba - control & $-4.32(-5.61 ;-3.03)$ & 0.000 & $-4.03(-5.32 ;-2.75)$ & 0.000 \\
\hline Act - control & $-6.43(-7.74 ;-5.13)$ & 0.000 & $-0.02(-1.60 ; 1.30)$ & 0.001 \\
\hline Ba-act & $2.11(0.84 ; 3.38)$ & 0.001 & $-4.01(-5.27 ;-2.74)$ & 0.000 \\
\hline \multicolumn{5}{|l|}{ Avoidant coping } \\
\hline Ba - control & $-16.11(-17.99 ;-14.23)$ & 0.000 & $-16.33(-17.76 ;-14.89)$ & 0.000 \\
\hline Act - control & $-14.46(-16.37 ;-12.56)$ & 0.000 & $-0.15(-1.60 ; 1.30)$ & 0.001 \\
\hline Ba - act & $-1.64(-3.49 ; 0.20)$ & 0.095 & $-16.17(-17.59 ;-14.76)$ & 0.000 \\
\hline \multicolumn{5}{|c|}{ Metacognitive beliefs } \\
\hline Ba - control & $-247.89(-263.39 ;-232.39)$ & 0.000 & $-250.31(-267.08 ;-233.54)$ & 0.000 \\
\hline Act - control & $-341.87(-357.60 ;-326.15)$ & 0.000 & $3.78(-13.22 ; 20.78)$ & 0.001 \\
\hline Ba-act & $93.98(78.70 ; 109.26)$ & 0.000 & $-245.09(-270.63 ;-237.56)$ & 0.000 \\
\hline \multicolumn{5}{|c|}{ Cognitive-attentional syndrome } \\
\hline Ba- control & $-268.33(-283.47 ;-253.19)$ & 0.000 & $-270.68(-287.88 ;-253.48)$ & 0.000 \\
\hline Act - control & $-362.78(-378.14 ;-347.43)$ & 0.000 & $3.59(-13.84 ; 21.04)$ & 0.001 \\
\hline Ba-act & $94.45(79.53 ; 109.37)$ & 0.000 & $-274.28(-291.24 ;-257.33)$ & 0.000 \\
\hline
\end{tabular}

Abbreviations: BA, behavioral activation; ACT, acceptance and commitment therapy; 95\% CI, 95\% confidence interval. 
liefs, rumination associated with concern, and hypervigilance (24), and the interruption of this cycle can disrupt this disorder. Regarding the higher effectiveness of ACT than BA, the rumination and the perception of negative emotions seem to be induced by high neural activity in the amygdala, hippocampus, cingulate, and dorsal cortex in patients suffering from depression (25). In other words, the more significant effect of ACT therapy on concerns and threat and metacognitive beliefs motivating worry can be underlined by the immediate sedative properties of this technique in relieving emotional stress posed by rumination and concerns in these patients (15).

Compared to the structured BA therapy targeting emotions, cognitions, and behaviors, the ACT treatment mostly focuses on desirable moods and emotional aspects of patients. It adopts techniques such as raising awareness and acceptance of negative emotions and bringing concentration and awareness to the now and the present to distract the mind and feelings of patients with depression from sadness, grief, emptiness, concerns, and rumination about imaginary threats. During the short term, it can have strong effects on reducing concerns and threat and metacognitive beliefs in patients. Given that the CAS- 1 test mostly evaluates factors that are adapted from ACT, therefore, this may be a reason for the difference between the results of the two treatments. The sustainable effect of BA after two months and the insignificant effect of the ACT technique during the same period can be attributed to the structure of the BA program. This is because this technique focuses on a systematic method, which concentrates not only on different dimensions of depression but also on the establishment of knowledge in patients regarding the defected cycle of their metacognitive patterns and avoidance behaviors. It also reconstructs behaviors and prescribes non-avoidant coping skills such as problem-oriented and positive emotion-oriented ones and the continuation and stabilization of new behavioral habits (26). This made its effect last for two months after the intervention. On the other hand, ACT mostly focuses on distracting patients from the present moment and mitigating the emotional effects such as sadness, grief, and emptiness in the present moment.

To explain this finding, fMRI has documented stronger connections in some areas of the brain belonging to the default network, such as the cingulate cortex in depressed patients, compared to healthy individuals $(27,28)$. This indicates the difficulty in regulating emotional, cognitive, and behavioral processing caused by the CAS severity in moderate to severe depression (29). Accordingly, reducing the burden of negative emotions, breaking the chains of rumination and concerns, and then modifying cognitive distortions by metacognitive interventions, providing a fo- cus on changing the mental structure, enhance the likelihood of long-term effectiveness of the techniques as nonpharmacological interventions.

\subsection{Limitations}

The present study was performed on patients with depression in Ahvaz city. Thus, caution should be observed in generalizing the results to other communities in different time and place situations due to different cultural conditions. Considering the lack of control on individual differences such as socioeconomic status and social support, it is suggested that similar studies examine larger sample sizes and more anxiety-mood disorders by controlling interfering variables so that the effect of each technique on each CAS component be assessed.

\subsection{Conclusions}

Behavioral activation and acceptance, and commitment therapy are effective in improving cognitiveattention syndrome in patients with depression. Therefore, these two therapies can be used as effective interventions in the treatment of patients with depression. Also, due to the greater effect of acceptance and commitment therapy on the cognitive-attention syndrome in patients with depression and the stability of behavioral activation after two months of follow-up in reducing cognitive-attention syndrome, it is suggested that these two treatments be performed for patients with depression.

\section{Footnotes}

Authors' Contribution: Sahar Amiri: Study concept and design, acquisition of data, analysis and interpretation of data, and statistical analysis. Parvin Ehteshamzadeh: Administrative, technical, and material support, and study supervision. Mohammad Reza Borna: Critical revision of the manuscript for important intellectual content.

Conflict of Interests: No conflict of interest is declared.

Ethical Approval: The study was approved by the Ethics Committee of Islamic Azad University, Ahvaz Branch (code: IR.IAU.AHVAZ.REC.1399.009).

Funding/Support: This study did not receive any funding.

Informed Consent: Questionnaires were filled out with the participants' satisfaction, and written informed consent was obtained from the participants in this study. 


\section{References}

1. Nobahar M, Hydarinia-Naieni Z, Ghorbani R. The prevalence of depression, anxiety, and stress and their association with vitamin $\mathrm{D}$ and estrogen levels in postmenopausal women in Semnan. Middle East J Rehabil Health Stud. 2019;6(4). doi: 10.5812/mejrh.91953.

2. Burkhouse KL, Jacobs RH, Peters AT, Ajilore O, Watkins ER, Langenecker SA. Neural correlates of rumination in adolescents with remitted major depressive disorder and healthy controls. Cogn Affect Behav Neurosci. 2017;17(2):394-405. doi: 10.3758/s13415-016-0486-4. [PubMed: 27921216]. [PubMed Central: PMC5366093].

3. Soleimani M, Mohammadkhani P, Dolatshahi B, Alizadeh H, Overmann KA, Coolidge FL. A comparative study of group behavioral activation and cognitive therapy in reducing subsyndromal anxiety and depressive symptoms. Iran J Psychiatry. 2015;10(2):71-8. [PubMed: 26884782]. [PubMed Central: PMC4752528].

4. Kowalski J, Wypych M, Marchewka A, Dragan M. Neural correlates of cognitive-attentional syndrome: An fMRI study on repetitive negative thinking induction and resting state functional connectivity. Front Psychol. 2019;10:648. doi: 10.3389/fpsyg.2019.00648. [PubMed: 30971987]. [PubMed Central: PMC6443848].

5. Wells A. Metacognitive therapy for anxiety and depression. Reprint edition ed. London, UK: The Guilford Press; 2009. 316 p.

6. Wisco BE. Depressive cognition: self-reference and depth of processing. Clin Psychol Rev.2009;29(4):382-92. doi:10.1016/j.cpr.2009.03.003. [PubMed: 19346043].

7. Giorgio JM, Sanflippo J, Kleiman E, Reilly D, Bender RE, Wagner CA, et al. An experiential avoidance conceptualization of depressive rumination: three tests of the model. Behav Res Ther. 2010;48(10):102131. doi: 10.1016/j.brat.2010.07.004. [PubMed: 20691426]. [PubMed Central: PMC3045819].

8. Kanter JW, Baruch DE, Gaynor ST. Acceptance and commitment therapy and behavioral activation for the treatment of depression: description and comparison. Behav Anal. 2006;29(2):161-85. doi: 10.1007/BF03392129. [PubMed: 22478462]. [PubMed Central: PMC2223147].

9. Dobson KS, Hollon SD, Dimidjian S, Schmaling KB, Kohlenberg RJ, Gallop RJ, et al. Randomized trial of behavioral activation, cognitive therapy, and antidepressant medication in the prevention of relapse and recurrence in major depression.J Consult Clin Psychol.2008;76(3):46877. doi: 10.1037/0022-006X.76.3.468. [PubMed: 18540740]. [PubMed Central: PMC2648513].

10. Takagaki K, Okamoto Y, Jinnin R, Mori A, Nishiyama Y, Yamamura $\mathrm{T}$, et al. Enduring effects of a 5-week behavioral activation program for subthreshold depression among late adolescents: an exploratory randomized controlled trial. Neuropsychiatr Dis Treat. 2018;14:263341. doi: 10.2147/NDT.S172385. [PubMed: 30349261]. [PubMed Central: PMC6186299].

11. Jafari D, Salehi M, Mohmmadkhani P. Comparison of the effectiveness of mindfulness-based cognitive therapy and behavioral activation treatment for depression in reducing symptoms, improving quality of life and decreasing suicidal thoughts in depressed individuals. Iran J Psychiatry Clin Psychol. 2015;20(4).

12. Teimourpour S, Akbari M, Hasani J. The evaluation of mechanism of effectiveness of behavioral activation therapy (ba) through cognitive flexibility and emotional flexibility on symptoms of women with major depressive disorders. J Cogn Psychol. 2019;6(4 p00819).

13. Bond FW, Dryden W. Handbook of brief cognitive behaviour therapy. 1st ed. Wiley; 2005. $330 \mathrm{p}$.

14. Heydari M, Masafi S, Jafari M, Saadat SH, Shahyad S. Effectiveness of acceptance and commitment therapy on anxiety and depression of razi psychiatric center staff. Open Access Maced J Med Sci. 2018;6(2):410-5. doi: 10.3889/oamjms.2018.064. [PubMed: 29531615]. [PubMed Central: PMC5839459].
15. Petkus AJ, M A, Wetherell JL. Acceptance and commitment therapy with older adults: Rationale and considerations. Cogn Behav Pract. 2013;20(1):47-56. doi: 10.1016/j.cbpra.2011.07.004. [PubMed: 26997859]. [PubMed Central: PMC4795910].

16. Kellett S, Simmonds-Buckley M, Bliss P, Waller G. Effectiveness of group behavioural activation for depression: A pilot study. Behav Cogn Psychother. 2017;45(4):401-18. doi: 10.1017/S1352465816000540. [PubMed: 28287065].

17. Szuhany KL, Otto MW. Efficacy evaluation of exercise as an augmentation strategy to brief behavioral activation treatment for depression: a randomized pilot trial. Cogn Behav Ther.2020;49(3):228-41. doi: 10.1080/16506073.2019.1641145. [PubMed: 31357916]. [PubMed Central: PMC6989384].

18. Babakhanzadeh S, Ahteshamzadeh P, Eftekharsaadi Z, Bakhtiyarpor $S$, Alipor A. The effectivenss of metacognitve therapy with emphasis for brain executive functions on cognitive-attentional syndrom and sense of coherence in anxious persons. Couns Cultur Psychother. 2019;10(38):195-220.

19. Melli G, Bailey R, Carraresi C, Poli A. Metacognitive beliefs as a predictor of health anxiety in a self-reporting Italian clinical sample. Clin Psychol Psychother. 2018;25(2):263-71. doi: 10.1002/cpp.2159. [PubMed: 29226504]

20. A.TjakJGL, Morina N, BoendermakerWJ, Topper M, Emmelkamp PMG Explicit and implicit attachment and the outcomes of acceptance and commitment therapy and cognitive behavioral therapy for depression. BMC Psychiatry. 2020;20(1):155. doi: 10.1186/s12888-020-02547-7. [PubMed: 32264845]. [PubMed Central: PMC7137238].

21. Kazemeyni M, Bakhtiari M, nouri M. Effectiveness of acceptance and commitment group therapy on postpartum depression and psychological flexibility. J Clin Nurs Midwife. 2018;6(4):20-31. eng.

22. Salmani B, Hasani J. Cognitive attentional syndrome(CAS) \& cognitive emotion regulation strategies: Transdiagnostic processes or diagnostic based on mood \& anxiety disorders. J Clin Psychol. 2013;5(3 (19)).

23. Kanter JW, Busch AM, Rusch LC. The CBT distinctive features series. Behavioral activation: Distinctive features. Routledge/Taylor \& Francis Group; 2009.

24. Groeneveld IF, van der Pas SL, Meesters JJL, Schuurman JM, van Meijeren-Pont W, Jagersma E, et al. Illness perceptions of stroke survivors: Predictors and changes over time - A 1year follow-up study. J Psychosom Res. 2019;116:54-61. doi: 10.1016/j.jpsychores.2018.10.019. [PubMed: 30654994].

25. Reavell J, Hopkinson M, Clarkesmith D, Lane DA. Effectiveness of cognitive behavioral therapy for depression and anxiety in patients with cardiovascular disease: A systematic review and meta-analysis. Psychosom Med. 2018;80(8):742-53. doi: 10.1097/PSY.0000000000000626. [PubMed: 30281027].

26. Rahbaran R, Karami R, Shahmohammadi M. The effectiveness of behavioral activation therapy on rhubarb and depression disorders in adolescents. Though Behav Clin Psychol. 2019;14(52):17-26.

27. Wells A, McNicol K, Reeves D, Salmon P, Davies L, Heagerty A, et al. Improving the effectiveness of psychological interventions for depression and anxiety in the cardiac rehabilitation pathway using group-based metacognitive therapy (PATHWAY Group MCT): study protocol for a randomised controlled trial. Trials. 2018;19(1):215. doi: 10.1186/s13063-018-2593-8. [PubMed: 29615092]. [PubMed Central: PMC5883514].

28. Keller AS, Leikauf JE, Holt-Gosselin B, Staveland BR, Williams LM. Correction: Paying attention to attention in depression. Transl Psychiatry. 2020;10(1):64. doi: 10.1038/s41398-020-0748-3. [PubMed: 32066703]. [PubMed Central: PMC7026409].

29. Berman MG, Misic B, Buschkuehl M, Kross E, Deldin PJ, Peltier $\mathrm{S}$, et al. Does resting-state connectivity reflect depressive rumination? A tale of two analyses. Neuroimage. 2014;103:267-79. doi: 10.1016/j.neuroimage.2014.09.027. [PubMed: 25264228]. 